\title{
Obstructive sleep apnea and risk of COVID-19 infection, hospitalization and respiratory failure
}

\author{
M. D. de Kruif ${ }^{1}$ - S. F. J. Voncken ${ }^{1}$ (D) - S. A. J. S. Laven ${ }^{1}$ • T. M. H. Feron ${ }^{1}$ - A. A. B. Kolfoort-Otte ${ }^{1}$
}

Received: 20 November 2020 / Revised: 20 November 2020 / Accepted: 11 December 2020 / Published online: 16 January 2021

(C) The Author(s), under exclusive licence to Springer Nature Switzerland AG part of Springer Nature 2021

Dear editor,

Maas et al. conducted a retrospective study to estimate the risk for coronavirus disease 2019 (COVID-19) infection in patients with obstructive sleep apnea (OSA) and to study the relationship between OSA and COVID-19 disease severity [1]. The researchers concluded that patients with OSA experienced an 8fold greater risk for COVID-19 in comparison to a control group derived from a large patient registry including data from 10 different hospitals. These results evoke a sense of alarm for patients with OSA, given the seriousness of the ongoing COVID-19 pandemic. However, we wish to express some concerns about the study.

Our primary concern is that the findings in the study do not match our expectations given that in a general population, the identified prevalence of OSA is 3-7\% [2]. Whereas the prevalence of $6.3 \%$ found by Maas et al. for OSA in patients with COVID-19 is well within this range, the prevalence in the control group was only $0.8 \%$. The authors do recognize that coding of administrative data is imprecise but they state that OSA was probably registered well by arguing that in a comparable clinical database, recording of OSA in Ottawa was $98 \%$ specific in comparison to a health administration database. However, the authors did not show a validation of the quality of their own database and do not offer an explanation for the unexpected low prevalence of OSA in the non-COVID-19 group.

A more detailed consideration of the non-COVID-19 group raises additional concerns. This control group was poorly characterized by the authors. The collected variables included sex, age (dichotomized at 50 years, for reasons not clarified), body mass index (BMI), race and two comorbidities (diabetes mellitus and hypertension). Data are presented for sex, age and race but not for BMI or comorbidities and the calculated odds ratio for the presence of OSA between COVID-19 and non-COVID-19

S. F. J. Voncken

susan.voncken@hotmail.com

1 Department of Pulmonology, Zuyderland Medical Centre, Henri Dunantstraat 5, 6419 PC Heerlen, Limburg, The Netherlands groups was not adjusted for these variables. In addition, socioeconomic factors have not been discussed, whereas it is known that a low socio-economic status is associated both with OSA and with COVID-19 [3, 4].

Furthermore, the authors claim that the registry included $5,544,884$ patient records from 10 hospitals but the history of this cohort is unclear, since it was stated that data were collected between January 1, 2020, and June 25, 2020. Because it is unlikely that this number of patients entered the hospitals within this study period, a significant amount of data is probably dated to earlier times. This may have given rise to a bias in the registration of OSA.

Overall, we recommend that the authors clarify their results further.

\section{Compliance with ethical standards}

Conflict of interest The authors declare that they have no competing interests.

\section{References}

1. Maas MB, Kim M, Malkani RG, Abbott SM, Zee PC (2020) Obstructive sleep apnea and risk of COVID-19 infection, hospitalization and respiratory failure. Sleep Breath:1-3. https://doi.org/10. 1007/s11325-020-02203-0

2. Punjabi NM (2008) The epidemiology of adult obstructive sleep apnea. Proc Am Thorac Soc 5(2):136-143

3. Guglielmi O, Lanteri P, Garbarino S (2019) Association between socioeconomic status, belonging to an ethnic minority and obstructive sleep apnea: a systematic review of the literature. Sleep Med 57: 100-106

4. Patel JA, Nielsen FBH, Badiani AA, Assi S, Unadkat VA, Patel B, Ravindrane R, Wardle H (2020) Poverty, inequality and COVID-19: the forgotten vulnerable. Public Health 183:110-111

Publisher's note Springer Nature remains neutral with regard to jurisdictional claims in published maps and institutional affiliations. 